Konsumaten-Ansatz als Werkzeugkasten zu verwenden, die dem Modellierer eine Heuristik zur Integration einer relativ einfachen Verhaltensdynamik in integrierte Modelle bietet. So kann der Konsumaten-Ansatz zur Simulation von gesellschaftlichen Prozessen und Gewohnheitsverhalten wie auch überlegtem Verhalten verwandt werden, um die dem Konsum von öffentlichen Gütern zugrunde liegende Verhaltensdynamik zu entschlüsseln und geeignete Managementstrategien für unsere öffentlichen Güter zu entwickeln.

(Übersetzung: Irene Siebcke)

\section{Anmerkungen}

(1) Dies wurde unlängst in einer Spezialausgabe der Zeitschrift Ecological Economics betont. Vgl. Janssen, M.A./ Jager, W.: The Human Actor in Ecological-Economic Models. Ecological Economics, Vol. 35, №. 3 (2000), S. 307-310.
(2) Der Beitrag basiert auf Jager, W./ Janssen, M.A./ de Vries, H.J.M./ de Greef, J./ Vlek C.A.J.: Behaviour in commons dilemmas: Homo economicus and Homo psychologicus in an ecological-economic model. Ecological Economics, Vol. 35, №. 3 (2000), S. 357-380. Wir danken Bert de Vries, Jodi de Greef und Charles Vlek für ihre Kooperation und Beiträge hierzu.

(3) Ein Überblick über dieses sich entwickelnde Gebiet findet sich bei Gilbert, G.N./ Troitzsch, K.G.: Simulation for the social scientist. Milton Keynes 1999.

(4) Dasgupta, P.S./ Heal G.M.: Economic theory and exhaustible resources. Welwyn et al., Cambridge University Press 1979.

(5) Vgl. Jager, W.: Modelling consumer behaviour. Dissertation, Universität Groningen 2000. Mehr Informationen über den Konsumaten-Ansatz finden sich im Internet unter http://go.to/consumats

(6) Vgl. De Greef, J./ De Vries, H.J.M.: Duurzame ontwikkeling als richtinggevend kader voor milieubeleid. RIVM, Bilthoven (NL) 1991.
(7) Max-Neef, M.: Development and human needs. In: Ekins, P./ Max-Neef, M. (Eds.): Real-life economics: Understanding wealth creation. London, New York 1992.

(8) Für eine detaillierte Modellanalyse siehe Jager et al. a.a.0. (Anm. 2).

\section{Die Autoren}

Dr. Wander Jager ist Assistenzprofessor an der Universität Groningen.

Kontakt: University of Groningen, Department of Marketing, PO Box 800, NL-9700 AV Groningen, E-mail: w.jager@bdk.rug.nl

Dr. Marco A. Janssen ist wissenschaftlicher Mitarbeiter an der Freien Universität Amsterdam.

Kontakt: Vrije Universiteit, Department of Spatial Economics, De Boelelaan 1105, NL-1081 HV Amsterdam, E-mail: m.janssen@econ.vu.nl.

\title{
Jenseits des Gleichgewichts
}

Für ein Verständnis der Mensch-Umwelt-Beziehungen, wie sie im Zentrum der Ökologischen Ökonomik stehen, bedarf es auch einer differenzierten Vorstellung von menschlichen Handlungsabläufen. Erst vor diesem Hintergrund können auch ökologisch motivierte Maßnahmen zur Veränderung des Verhaltens sinnvoll beurteilt werden. Neve Modellierungsansätze bieten hierzu die Möglichkeit. Vorgestellt werden drei Bausteine, mit denen sich ein Lern- oder Regeländerungsverhalten der Akteure, komplexere Interaktionsstrukturen sowie das Handeln verschiedenartiger Akteure in einer räumlichen Umwelt abbilden lassen.

D ie Diskussion um eine Nachhaltige Entwicklung konzentrierte sich lange auf normative Fragen: Welche Arten und Systemfunktionen (Klima, Wasserhaushalt etc.) sind zu erhalten und in welchem Maße? Es wurden unzählige Indikatoren, Grenzwerte und Eingriffsregelungen entwickelt und diskutiert, wie solche Nutzungsgrenzen am besten durchsetzbar wären: ob über Verbote, Steuern, Umweltzertifikate, internationale Abkommen oder genetische Eigentumsrechte. Für eine genauere Einschätzung der Angriffsflächen und Wirkung solcher Maßnahmen ist es jedoch wichtig, zunächst einmal beschreiben zu können, welche Motive, Informationen und Formen der Informationsgewinnung und -verarbeitung die Menschen bei der Nutzung der natürlichen Umwelt derzeit bestimmen und wie sich ihr
Handeln unter Einfluss dieser Faktoren verändert - oder verändern lässt. Regelungen und Lenkungsmaßnahmen können nur greifen, wenn die Betroffenen zumindest einigermaßen so auf sie reagieren, wie erwartet.

\section{Neve Perspektiven für die Ökologische Ökonomik}

Nachdem zunächst eine zwar kritische Haltung gegenüber traditionellen umweltökonomischen Modellen vorherrschte, aber von Seiten der Ökologischen Ökonomik wenig eigene Modellierungsansätze vorgeschlagen wurden, ist dort in den letzten Jahren die Erkenntnis gewachsen, dass einer realistischeren Abbildung menschlichen Handelns in ökologisch-ökonomischen Modellen große Bedeutung zukommt. Auslöser für diesen Wandel sind auch die zunehmende Leistungsfähigkeit von Computern und die For- schungsergebnisse anderer Fachdisziplinen, die Möglichkeiten neuer Formen der Modellierung eröffneten.

Die Neuerungen selbst sind vor allem in drei Bereichen angesiedelt. Zum einen wird versucht, ökologische Abläufe differenzierter zu modellieren, als dies in den meisten umwelt- und ressourcenökonomischen Modellen der Fall ist. Peterson weist zum Beispiel mit Bezug auf Erkenntnisse der Ökosystemforschung darauf hin, dass Ökosysteme wiederkehrende Phasen von Strukturaufbau, Wachstum und Verfall durchlaufen, in denen sie unterschiedlich stabil sind und entsprechend unterschiedlich sensibel auf menschliche Eingriffe reagieren (1). Weitaus häufiger lassen sich allerdings Modelle finden, in denen ein anderer wesentlicher Aspekt ökologisch-ökonomischer Zusammenhänge anspruchsvoller ausgearbeitet wird als in standardökonomischen Modellen: menschliche Entscheidungsabläufe unter der Voraussetzung beschränkter Rationalität (vgl. den Beitrag von Beckenbacb). Eine dritte wesentliche Erkenntnis besteht schließlich in der Anerkennung der Bedeutung, die Interaktion der Wirtschaftsakteure explizit abzubilden, da sich viele Phänomene nur so erklären lassen. Beispiele sind Moden, Normen, Lernen voneinander sowie gruppenspezifische Ansprüche und Bedürfnisse, die letztlich alle auf die Art und Intensität der Umwelt- und Ressourcennutzung einwirken.

Die Vielfalt neuer Modelle (2) lässt sich nicht immer eindeutig bestimmten Modelltypen zuordnen, sie beinhalten jedoch häufig gewisse Grundelemente, die als Bausteine einer differenzierte- 
ren Modellierung menschlicher Handlungsabläufe angesehen werden können. Je nach Modell werden einzelne dieser Bausteine verwendet oder mehrere Elemente kombiniert. Im Folgenden werden drei solcher Bausteine vorgestellt, mit denen sich ein Lern- oder Regeländerungsverhalten der Akteure, räumliche Interaktionen und komplexere Interaktionsstrukturen sowie das Agieren in einer räumlichen Umwelt abbilden lassen:

- Die Modellierung von Lernen auf der Basis Genetischer Algorithmen,

- Zelluläre Automaten zur Abbildung nachbarschaftlicher Interaktionen und

- Multi-Agenten-Systeme, die eine Verknüpfung von Verhaltensalgorithmen und räumlichen Aspekten erlauben und die ersten beiden Elemente beinhalten können.

\section{- Modellierung von Lernen auf der Basis Genetischer Algorithmen}

Wenn Wirtschaftsakteure nicht perfekt über ihre Umwelt informiert sind, können sie entweder einfachen Handlungsregeln folgen (3) oder sie müssen lernen. Wie erfolgt Lernen? Zunächst wird eine bestimmte Verhaltensweise ausprobiert und ihr Erfolg begutachtet. Mangels eines absoluten Erfolgsmaßstabs - wirkliche Menschen kennen in der Regel nicht das Optimum, das aus einer Situation herauszuholen sein könnte - bietet sich ein Vergleich mit anderen Akteuren oder mit einem persönlichen Anspruchsniveau an. Bei Unzufriedenheit kann der Wunsch nach Veränderung entstehen. Häufig sind es allerdings auch erfolgreiche Akteure, die ihre marktführende Position ausbauen wollen und nach weiteren Neuerungen suchen, um ihren Vorsprung nicht zu gefährden. Der eigentliche Lernvorgang kann durch Imitation oder Innovation erfolgen. Die zunächst naheliegendste Maßnahme ist, sich umzuschauen, wer erfolgreicher war als man selbst und dessen Strategie zu kopieren: Imitation. Innovation, also die Entwicklung von Neuem, kann zwei Elemente beinhalten. Entweder werden zufällige Veränderungen an bestehenden Strategien oder Technologien vorgenommen und ausprobiert, welche Variante die geeignetste ist, oder alte Elemente werden neu verknüpft. Beide Lernformen erfordern häufig eine zumindest indirekte Interaktion mit anderen, weil es deren Produkte, Strategien oder Technologien sind, die imitiert werden oder die als Rohmaterial für die Entwicklung neuer Varianten und Rekombinationen dienen. In der Vergangenheit erfolgreiche Varianten werden dabei mit höherer Wahrscheinlichkeit imitiert oder weiterentwikkelt.

Viele der Kernelemente eines solchen Lernablaufs sind dem biologischen Evolutionsprozess nicht unähnlich. Dies und die Tatsache, dass ein Evolutionsbiologe ein einfaches Computermodell entwickelt hat, mit dem sich ein Evolutionsprozess über Selektion, Reproduktion, Rekombination und Mutation simulieren lässt, haben dazu geführt, dass Ökonomen bei der Suche nach einer geeigneten Abbildung von Lernprozessen auf den von Holland entwickelten Genetischen Algorithmus (GA) zurückgegriffen haben (4).

Entscheidungsregeln oder Produktionsprozesse werden bei diesem Verfahren - analog zum genetischen Material - in Zeichenketten, so genannten Strings, ausgedrückt, aus denen sich Handlungsoder Produktionsweisen ergeben. Einzelne Stringabschnitte könnten etwa für verschiedene Produktionsfaktoren und deren Einsatzmengen stehen. Diese Entscheidung wird auf dem Markt wirksam, indem eine bestimmte Produktmenge angeboten wird. Die aktuelle Marktlage bestimmt, wie sich die Entscheidung auf Gewinne oder Absatzchancen auswirkt. Die Marktreaktion wird dann wiederum von den Akteuren bewertet. Kriterien hierfür können zum Beispiel ein möglichst hoher Gewinn oder Marktanteil oder auch das Ziel einer nachhaltigen Ressourcennutzung sein. Im Vergleich mit anderen ergibt sich, wer das Zielkriterium wie gut erreicht hat. Der Erfolg ist ausschlaggebend für den Selektionsprozess. Erfolgreiche Strategien werden mit höherer Wahrscheinlichkeit ausgewählt. Sie werden anschließend entweder unverändert in die nächste Periode übernommen (Replikation oder Imitation) oder, analog zum genetischen Cross-over, zu neuen Varianten rekombiniert bzw. durch Experimente oder Fehler, die den biologischen Mutationen entsprechen, verändert (Innovation).

Die ursprünglichen Genetischen Algorithmen finden selbst in komplizierten Umgebungen meist sehr schnell gute Lösungen (vgl. Abbildungen 1 und 2). Aus diesem Grund wurden GA, die eigentlich als beschreibendes Verfahren entwickelt wurden, häufig als Problemlösungsverfahren eingesetzt. Im ökonomischen Bereich werden sie beispielsweise zur Optimierung von Transportnetzen, zur Auslastung von Leitungen oder in der Lagerhaltung verwendet.

Diese Verwendungen des GA unterscheiden sich grundsätzlich von ihrem Einsatz als Lernmodelle, wo sie wieder beschreibenden Charakter haben.

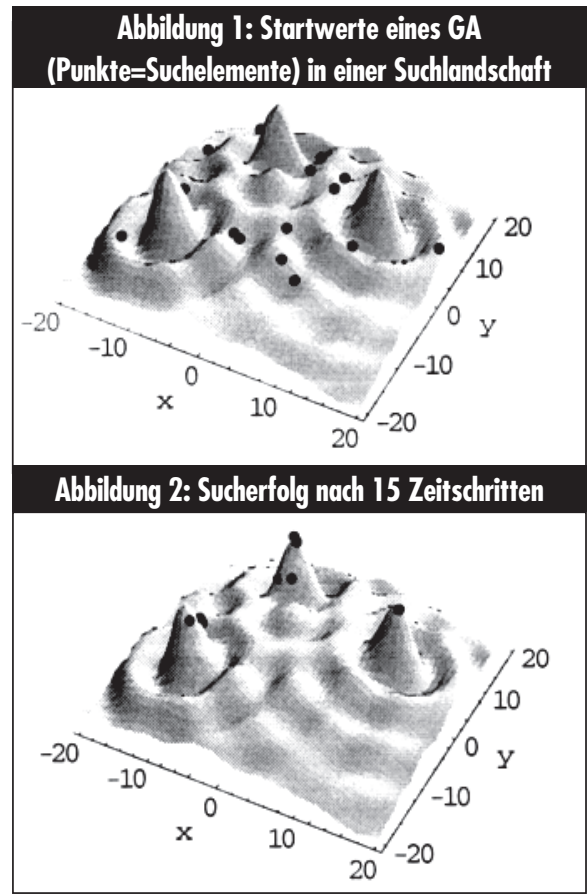

Quelle: eigene Darstellung

Der wesentliche Unterschied liegt in der Gestaltung der Zielfunktion und des Selektionsmechanismus, die für die Entwicklung neuer Strategien zentral sind. In einem Optimierungsmodell existieren externe Vorgaben. Derjenige, der dem vorgegebenen Ziel am nächsten kommt, ist auch tatsächlich der Beste. Lernen erfolgt dagegen, indem selbst gesteckte Ziele verfolgt werden. Möglicherweise sind die Ziele mancher Wirtschaftsakteure nicht geeignet, ein langfristiges Überleben am Markt zu sichern. Möglicherweise merken sie dies aber nicht und versuchen trotzdem weiter, kurzfristig Gewinne zu maximieren, bis alle gemeinsam Verluste machen - etwa nachdem eine Ressource erschöpft oder übernutzt wurde.

Bei der Gestaltung von Lernmodellen ist sorgfältig darauf zu achten, was die abzubildenden Akteure tatsächlich für Ziele verfolgen und welche Informationen ihnen bei ihrem Lernprozess zur Verfuigung stehen. Aus derartigen Modellen können dann sowohl Schlussfolgerungen darüber gezogen werden, welche Lernziele und Informationen zu welcher Umweltsituation führen, als auch an welcher Stelle Informationspolitik betrieben werden müsste, um eine nachhaltige Entwicklung wahrscheinlicher zu machen.

GA wurden in der Ökonomik bereits zur Abbildung verschiedener Lernsituationen eingesetzt. In der Ökologischen Ökonomik existieren bisher Modelle zur Verhaltensanpassung von Entscheidungsträgern der Klimaschutzpolitik (5), zum Vergleich von Lernstrategien mit schrittweisem 
und sprunghaftem Suchverhalten bei der Ressourcennutzung (6) und zur Bewirtschaftung regenerativer Ressourcen unter Gewinnorientierung oder Nachhaltigkeitsanspruch (7). Daraus resultierten unter anderem neue Erkenntnisse über die Schwierigkeiten menschlicher Akteure bei der Steuerung bereits relativ einfacher nichtlinearer ökologisch-ökonomischer Systeme und über die Vereinbarkeit von ökologischer und ökonomischer Nachhaltigkeit.

\section{Zelluläre Automaten}

Zelluläre Automaten (ZA) stellen eine Möglichkeit dar, die Beeinflussung eines Individuums durch seine Nachbarn abzubilden. Man kann sich einen ZA wie ein Schachbrett oder Karopapier vorstellen, dessen Kästchen entweder bewohnt oder unbewohnt sind und dessen Bewohner verschiedene Eigenschaften aufweisen können - wie reich oder arm zu sein, umweltbewusst oder nicht zu sein. Sowohl die Eigenschaften, als auch die Tatsache, ob ein Bewohner auf seinem Kästchen bleibt, umzieht oder eventuell auch stirbt bzw. aus einem Wirtschaftssektor ausscheidet, hängen von den Bedingungen in seiner Nachbarschaft $a b$. Wie diese Nachbarschaft räumlich abgegrenzt wird, ist Definitionssache.

Eine einfache Regel könnte beispielsweise lauten: „Kaufe keine Ökoprodukte, so lange die Mehrheit Deiner Nachbarn dies nicht tut." Mit solchen Modellen lässt sich anschaulich untersuchen, ob und wie sich bestimmte Einstellungen oder Produkte über lokale Interaktionen ausbreiten. Abbildung 3 ist ein Beispiel für einen solchen Anpassungsvorgang. Ausgehend von einem gleichverteilten anfänglichen Käuferverhalten passen sich die Akteure so an ihre jeweiligen Nachbarn an, dass sich ein Verhaltenstyp weitgehend durchsetzt. Die Käufer von Ökoprodukten können nur in einzelnen Stadtvierteln ,überleben“, dort überzeugen sie aber auch vorher nicht Kaufwillige.
Weisbuch et al. haben zum Beispiel die Kaufentscheidungen für Autos mit und ohne Katalysator in einem ZA modelliert (8). Die Entscheidung hängt von der eigenen Voreinstellung der Akteure, ihren Erfahrungen mit dem einen oder anderen Autotyp, der Zufriedenheit ihrer Nachbarn mit ihren jeweiligen Autos und von der Verschmutzung in ihrer Gegend ab. Dabei stellte sich heraus, dass unter diesen Voraussetzungen einer nur lokalen Informationsausbreitung die Zahlungsbereitschaft der Käufer für den Katalysator nur einen Bruchteil der Kosten der durch den Katalysator vermiedenen Umweltverschmutzung ausmacht, wohingegen unter Voraussetzung vollständiger Rationalität diese Zahlungsbereitschaft gerade den Betrag der vermiedenen Verschmutzungskosten erreichen müsste. Ein weiteres Anwendungsfeld ist die Modellierung der Veränderung von Siedlungsstrukturen in Abhängigkeit von den in der näheren Umgebung zu findenden Interaktionspartnern wie Kunden, Handelspartnern oder Gleichgesinnten.

\section{- Multi-Agenten-Systeme}

Multi-Agenten-Systeme (MAS) bilden den umfassendsten und flexibelsten der hier dargestellten Modellierungsansätze.

Im Mittelpunkt dieses Ansatzes steht die explizite Abbildung der einzelnen Akteure eines realen ökologisch-ökonomischen Systems sowie von deren Umwelt im Modell. Die Elemente eines MAS sind im einzelnen:

aktive, autonom handelnde Agenten,

passive Objekte (Umwelt),

- Beziehungen zwischen den Agenten untereinander,

- Beziehungen zwischen den Agenten und den Objekten der Umwelt sowie

- Beziehungen zwischen Objekten der Umwelt untereinander.

\section{Abbildung 3: Anpassung an das Mehrheitsverhalten der Nachbarn in einem ZA}

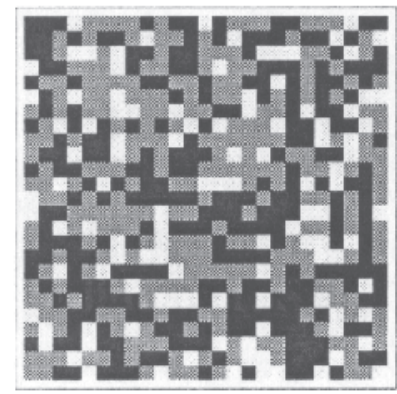

Ausgangszustand

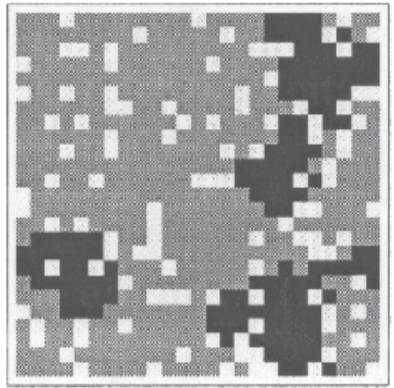

Endzustand

Quelle: eigene Darstellung

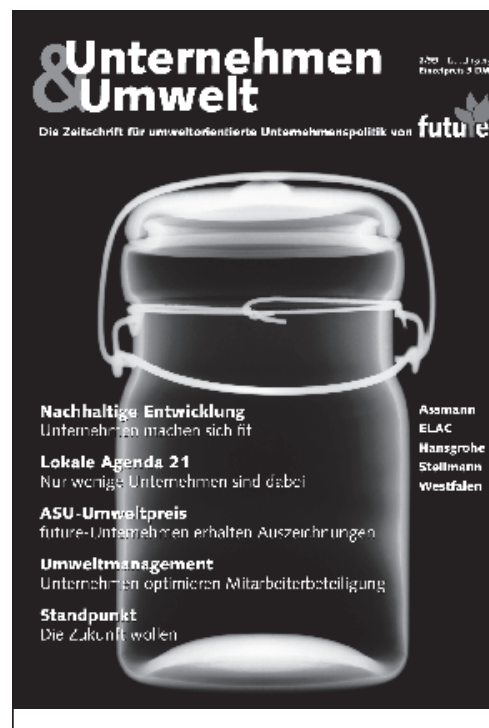

\section{Unternehmen \& Umwelt}

- ist die Zeitschrift für umweltorientierte Unternehmenspolitik von future e.V.,

- berichtet über den neuesten Stand zum betrieblichen Umweltmanagement in Theorie und Praxis,

- bietet vier Mal im Jahr ein Schwerpunktthema,

Unternehmensbeispiele, Literaturund Veranstaltungshinweise und aktuelle future-Nachrichten.

Zum Beispiel Heft 2/99:

\section{Nachhaltigkeit}

- Nachhaltige Entwicklung: Unternehmen machen sich fit.

- Lokale Agenda 21: Nur wenige Unternehmen sind dabei.

- Betrieblicher Umweltschutz: Assman, ELAC, Hansgrohe, Steilmann.

- Umweltmanagement: Unternehmen optimieren die Mitarbeiterbeteiligung.

Fordern Sie Ihr kostenloses

Probeexemplar an:

Fon: 0234 - 97995 - 13

Fax: 0234 - 97995 - 14

future e.V. Büro Bochum

Am Varenholt 123

44797 Bochum

email: future@umis.de 
Sowohl die Agenten als auch die Objekte sind in ein räumliches Bezugssystem eingebettet.

Charakteristisch für MAS ist die explizite Abbildung des inneren Zustands, der kognitiven Prozesse im Innern der Akteure (Wahrnehmung, Informationsverarbeitung, Gedächtnis, Lernen) sowie der Interaktionen (Informationsaustausch); diese Elemente spielen eine zentrale Rolle.

Da sich der innere Zustand eines Akteurs im Laufe der Zeit verändert, kann es passieren, dass ein und derselbe Akteur auf das gleiche äußere Ereignis zu verschiedenen Zeitpunkten verschieden reagiert. Daraus resultiert eine Pfadabhängigkeit des Systemverhaltens, das heißt das langfristige Verhalten des Gesamtsystems hängt auch von der Geschichte seiner Elemente ab.

Die Untersuchungsperspektive bei einem MAS geht ,,von unten nach oben“: Eine Grundannahme ist, dass die Komplexität des Systems durch Interaktion einfacherer Elemente zustande kommt; es wird also versucht, auf der Makroebene beobachtbare Phänomene wie die Übernutzung einer regenerierbaren Ressource oder urbane Segregation durch das Zusammenwirken von Prozessen auf Mikroebene, nämlich der Informationsverarbeitung durch die Akteure und der Interaktion zwischen ihnen, zu erklären. So wird es möglich, die endogene Dynamik eines komplexen Systems in ihrer zeitlichen und räumlichen Dimension zu analysieren - die Dynamik, die das System durch die Wechselwirkung seiner Elemente aus sich selbst heraus entwickelt.

Die klassische Umwelt- und Ressourcenökonomik sieht sich zwei wesentlichen Problemen gegenüber: zum einen dem Heterogenitätsproblem, das darin begründet liegt, dass die Akteure individuell unterschiedliche Merkmalsausprägungen besitzen, und zum anderen dem Interdependenzproblem, das sich aus der gegenseitigen Beeinflussung der Handlungen der einzelnen Akteure ergibt. Diese Probleme sind im Rahmen traditioneller Modelle nicht behandelbar, da diese Modelle mit aggregierten, auf die Population als Ganzes bezogenen Größen wie soziale Diskontrate oder Volkseinkommen operieren und Individuen als solche darin nicht vorkommen. Erst durch die Modellierung mit Hilfe von MAS werden diese beiden Probleme einer Untersuchung zugänglich; gerade die Möglichkeit der Modellierung heterogener Populationen von Agenten und ihrer vielfältigen Interaktionen ist eine wesentliche Stärke dieses Ansatzes (vgl. den Beitrag von Jager/Janssen).

Im Unterschied zu Zellulären Automaten können bei MAS Interaktionen auch in nicht räumlichen
Dimensionen von Lokalität stattfinden. Es können also Akteure miteinander interagieren, die nicht räumlich benachbart sind, sondern zum Beispiel durch funktionale (etwa Austausch von Waren oder Dienstleistungen) oder soziale Zusammenhänge (Bekanntschaft, Zugehörigkeit zu einer bestimmten sozialen Gruppe) zueinander in Beziehung stehen. Diese verschiedenen Dimensionen von Lokalität können in ein und dem selben Modell kombiniert werden. Darüber hinaus sind für jede dieser Formen lokaler Interaktion unterschiedliche Reichweiten möglich. Beispielsweise ist ein Modell konstruierbar, in dem ein Agent bezüglich der Wahrnehmung seiner Umwelt über ein Sichtfeld verfügt, das alle Zellen im Abstand von höchstens drei Kantenlängen der Zellen umfasst; dieser Agent könnte aber nur mit solchen Agenten Handel treiben, die sich in ihm unmittelbar benachbarten Zellen aufhalten, und er könnte dennoch seinen eigenen wirtschaftlichen Erfolg mit dem Erfolg seiner Bekannten vergleichen, die räumlich beliebig verstreut sind.

MAS ermöglichen auf der Mikroebene die Simulation ,intelligenter“ Akteure mit kognitiven Fähigkeiten wie Lernen, Bewertung und Evolution von Verhaltensstrategien und können dadurch zur Erklärung komplexer Phänomene auf der Makroebene beitragen. Epstein und Axtell haben mit relativ einfachen Verhaltensregeln für die Agenten komplexe sozioökonomische Prozesse wie Handel, Bildung sozialer Netzwerke, Seuchenausbreitung, Entstehung verschiedener Kulturen und deren gegenseitige Beeinflussung nachbilden können (9).

\section{- Fazit}

Die normativen Aussagen, die in einem früheren Entwicklungsstadium der Ökologischen Ökonomik im Vordergrund standen, etwa das Nachhaltigkeitspostulat oder andere umweltpolitische Forderungen, treten hier zurïck zugunsten des Ziels einer realistischen Nachbildung bzw. Simulation der komplexen Interdependenz der ökologischen und ökonomischen Systeme. Die Simulation bietet die Chance, die Realisierbarkeit solcher Forderungen zu prüfen und Handlungserfordernisse für ihre praktische Umsetzung zu spezifizieren (10). Die Verwendung des MAS-Ansatzes in der Ökologischen Ökonomik bietet realitätsnähere Mittel, um beispielsweise

sowohl erwünschte als auch nicht erwünschte Wirkungen neuer Produkte auf ökologisch-ökonomische Systeme vorherzusagen;
- Maßstäbe zu liefern, um den Sinn oder die Treffsicherheit politischer Maßnahmen im Hinblick auf zuvor gesetzte Umweltziele zu beurteilen;

die Marktchancen nichttechnischer ökologischer Innovationen (Produkt-, Funktions-, Bedürfnisinnovationen) abzuschätzen.

\section{Anmerkungen}

(1) Peterson, G.: Political ecology and ecological resilience: An introduction of human and ecological dynamics. In: Ecological Economics Vol. 35 (2000), №. 3, S. 323-336.

(2) Vgl. neben den weiteren Anmerkungen auch die Beitröge in der Sonderausgabe der Ecological Economics, Vol. 35, №. 3 (2000) zum Thema "The human Actor in EcologicalEconomic Models".

(3) Für entsprechende Modelle vgl. z.B. Allen, P.M./

McGlade, J.M.: Modelling complex human systems: A fisheries example. In: European Journal of Operational Research, Vol. 30 (1987), S. 147-167; Geisendorf, S.: Evolutorische Ökologische Ökonomie, Marburg 2001, Kap. 4.

(4) Holland, J.H.: Adaptation in Natural and Artificial Systems. Ann Arbor 1975.

(5) Janssen, M.A./ Vries, B. de: The battle of perspectives: a multi-agent model with adaptive responses to climate change. In: Ecological Economics, Vol. 26 (1998), S. 43-65.

(6) Beckenbach, F.: Beschränkte Rationalität und Systemkomplexität: Ein Beitrag zur Ökologischen Ökonomik, erscheint bei Metropolis.

(7) Geisendorf, S. a.a.0. (Anm. 3)

(8) Weisbuch, G./ Gutowitz, H./ Duchateau-Nguyen, G.: Information contagion and the economics of pollution. In: Journal of Economic Behaviour and Organization, Vol. 29 (1996), S. 389-407.

(9) Epstein, J.M./ Axtell, R.: Growing Artificial Societies: Social Science from the bottom up. Cambridge (Mass.) 1996.

(10) Vgl. z.B. Beckenbach, F.: Ressourcengewinnung und Marktkoordination in einem Multi-Agenten-System. In: Lehmann-Waffenschmidt, M.: Beiträge zur Evolutorischen Ökonomik, Band V. Berlin 2001.

\section{Die Autorlnnen}

Dr. Sylvie Geisendorf und Ramón Briegel sind wissenschaftliche MitarbeiterInnen an der Universität Kassel.

Kontakt: Universität Gesamthochschule Kassel, FB Wirtschaftswissenschaften, Nora-Platiel Str. 4, 34109 Kassel, E-mail: sylvie_g@hrz.uni-kassel.de, 
(c) 20I0 Authors; licensee IÖW and oekom verlag. This is an article distributed under the terms of the Creative Commons Attribution Non-Commercial No Derivates License (http://creativecommons.org/licenses/by-nc-nd/3.o/), which permits unrestricted use, distribution, and reproduction in any medium, provided the original work is properly cited. 